\title{
Contemporary agents in the management of metastatic castration-resistant prostate cancer
}

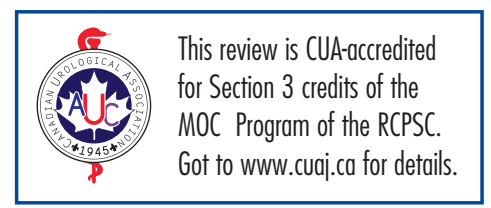

\author{
Anil Kapoor, MD, ${ }^{1,2}$ Christopher Wu, MD, ${ }^{1,2}$ Bobby Shayegan, MD, ${ }^{1,2}$ Adrian P. Rybak, MD ${ }^{1,2}$ \\ 'McMaster Institute of Urology, Division of Urology, Department of Surgery, McMaster University, Hamilton, ON, Canada; 25 t. Joseph's Hospital, Hamilton, ON, Canada
}

Cite as: Can Urol Assoc J 2016;10(11-12):E414-23. http://dx.doi.org/10.5489/cuaj.4112 Published online December 12, 2016.

\section{Abstract}

Docetaxel-based chemotherapy has been the standard of care for metastatic castration-resistant prostate cancer (mCRPC) since 2004. Over the past few years, there has been a significant paradigm shift in the treatment landscape of this disease. A deeper understanding of prostate cancer biology, along with the development of novel agents has created hope towards treating chemotherapy-naïve and resistant disease. Following the implementation of docetaxel as the first-line therapy for mCRPC, five novel therapies have demonstrated survival benefit in MCRPC. Cabazitaxel, abiraterone acetate, and enzalutamide are three agents recently approved for the treatment of mCRPC, having shown overall survival benefit in patients previously treated with docetaxel, while both abiraterone acetate and enzalutamide have also shown promise in the pre-docetaxel setting. Sipuleucel-T has shown overall survival benefit in asymptomatic mCRPC, while radium-223 provides survival benefit to patients with mCRPC who are symptomatic from their skeletal metastases in both docetaxel-naïve patients and post-docetaxel patients. Denosumab, an anti-RANKL antibody, has been approved for the prevention of skeletal-related events in patients with prostate cancer bone metastases. This review examines the phase 3 trials supporting the use of theses novel agents in the treatment of mCRPC. While these agents provide incremental increases in patient survival, further study to determine the best choice, combination, and/or sequencing of administration is still necessary.

\section{Introduction}

Prostate cancer $(\mathrm{PCa})$ is the most common male malignancy and second leading cause of cancer-related deaths in males in the Western world, with $24 \%$ and $27 \%$ of all new male cancer cases estimated to occur in 2014 in Canada and the U.S., respectively. ${ }^{1,2}$ PCa progresses from an androgendependent disease to castration-resistant (hormone-refractory) prostate cancer (CRPC), the major factor contributing to PCa-related death. ${ }^{3,4}$ Since the landmark discovery charac- terizing the dependence of PCa on androgens for growth and survival, ${ }^{5}$ androgen-deprivation therapy (ADT) has become the primary therapy for late-stage or disseminated PCa. ADT - which includes surgical castration (orchiectomy), chemical castration (luteinizing hormone-releasing hormone [LHRH] agonists or antagonists) and/or anti-androgen treatment to reduce serum testosterone levels to a minimum $(<50$ $\mathrm{ng} / \mathrm{dl})^{6}$ - demonstrates a good initial response in androgensensitive $\mathrm{PCa}$, including a significant biochemical response (80-90\% serum prostate-specific antigen [PSA] reduction) and clinical regression of the disease. Despite this initial response, ADT has been found to provide a survival advantage of only several months compared to control treatment, ${ }^{7,8}$ with eventual biochemical recurrence manifesting in most patients.

According to recent Canadian and American Urological Association guidelines, ${ }^{9,10}$ continued observation while maintaining ADT is recommended in patients with CRPC and non-metastatic disease, as other treatments have not been shown to prolong survival in these patients. If patients are unwilling to accept observation, secondary hormonal manipulation may be considered, which includes complete androgen blockade with the administration of first-generation anti-androgens (flutamide, bicalutamide, nilutamide). ${ }^{11}$ Once a patient develops detectable macroscopic metastatic disease while presenting castrate levels of testosterone following ADT, they are considered to have metastatic CRPC (mCRPC). Patients with mCRPC have a poor prognosis and have an expected survival of approximately 18-20 months. ${ }^{12,13}$

Several mechanisms are believed to lead to CRPC development: 1) persistence of intratumoural androgens due to in situ steroidogenesis ${ }^{14}$ or biosynthesis from adrenal glands and testes; ${ }^{15}$ 2) wild-type androgen receptor (AR) amplification and/or protein overexpression; ${ }^{16,17} 3$ ) constitutive activation of AR signaling and/or AR-targeted transcription due to AR mutation ${ }^{18}$ or post-translational modification; ${ }^{19} 4$ ) activation of other ligand-bound receptors, including receptor tyrosine kinases, ${ }^{20}$ and their downstream pro-survival signaling pathways; and 5) the presence of prostate cancer 
stem cells that initiate tumour formation and survive independently of androgen, subsequently sustaining tumourigenesis by promoting disease relapse following therapy and colonizing in foreign tissues. ${ }^{21}$

Identification and characterization of molecular targets leading to prostate tumour growth, despite an androgendepleted or androgen-independent cell state, has resulted in a significant number of new U.S. Food and Drug Administration (FDA) and Health Canada-approved therapeutic agents for mCRPC that offer an overall survival (OS) advantage. Although guidelines exist to help direct physicians in the sequencing options for therapeutic agent administration based on symptoms and performance status, alternative approaches to PCa treatment after therapy failure remain a challenge, especially with known crossresistance among these novel agents. ${ }^{22-24}$ This article will review the current literature on the treatment of mCRPC with special focus on immunotherapies, novel chemotherapeutic agents, hormonal therapies, and radiopharmaceuticals. A better understanding of the mechanisms and sites of action of these therapies (Fig. 1) will better assist physicians in making informed therapeutic decisions. This is of increasing importance, as there is paucity of data on the sequence of administration of these agents in the treatment of mCRPC.

\section{Treatment of $\mathrm{mCRPC}$ without symptoms}

\section{Sipuleucel-T: Immunotherapy for mCRPC treatment}

Sipuleucel-T is an autologous dendritic cell vaccine that involves ex vivo activation of peripheral blood mononuclear (PBMN) cells with a recombinant fusion protein (PA2024) consisting of prostatic acid phosphatase (PAP), a protein found in $95 \%$ of prostate cancers, and granulocyte-macrophage colony-stimulating factor (GM-CSF). PBMN cells are first obtained through leukapheresis, incubated with PA2024, and subsequently re-infused into patients in order to produce an immune response to PAP-expressing PCa cells. ${ }^{25}$

Sipuleucel-T was evaluated in two initial randomized, placebo-controlled, phase 3 trials (D9901 and D9902A) that were identical in design, allowing for a combined analysis

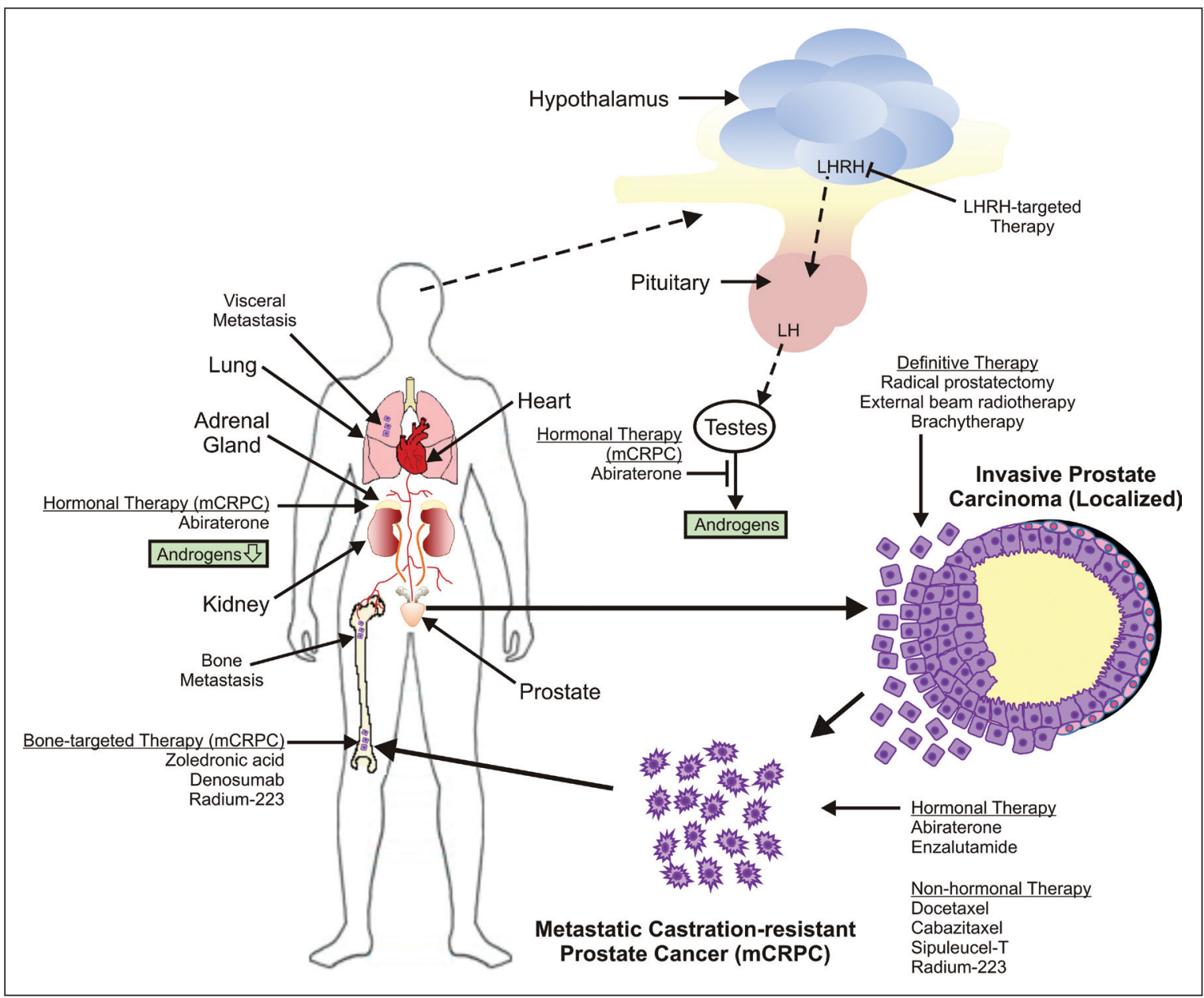

Fig. 1. Treatment options for localized and metastatic prostate cancer and the sites of action of agents used to treat metastatic castration-resistant prostate cancer. LH: luteinizing hormone; LHRH: luteinizing hormone-releasing hormone. 
of results. ${ }^{25,26}$ A total of 225 patients with asymptomatic mCRPC were enrolled and randomized $(2: 1)$ to sipuleucel$\mathrm{T}$ or placebo, administered as three intravenous infusions (each two weeks apart) with the primary objective being time to disease progression; OS was the secondary endpoint. The combined results of these trials showed a non-significant trend towards delay in time to disease progression for the sipuleucel-T treatment arm vs. placebo (11.1 vs. 9.7 weeks, respectively; $p=0.111$ ). However, a significant improvement in OS was observed in the sipuleucel-T treatment arm over placebo, with a median 4.3-month increase in survival (23.2 vs. 18.9 months, respectively; $p=0.011$ ). The disparity between time to disease progression and OS is believed to be due to the length of time needed to generate a maximal immune response to therapy. In some patients, maximal response may not occur until 12 weeks after therapy initiation, whereas the median time to progression in these studies was $10-12$ weeks, ${ }^{27}$ suggesting that the progression outcome may already have been reached by the time the therapy reached maximal efficacy.

The OS benefit seen in the initial phase 3 trials was further confirmed by the IMPACT study. The IMPACT trial was a double-blind, placebo-controlled, randomized, multicentre phase 3 trial in patients with asymptomatic or minimally symptomatic mCRPC. Eligible subjects for this trial had Eastern Cooperative Oncology group (ECOG) performance status of $\leq 1$, no visible visceral metastases, no treatment within 28 days prior to entry with systemic corticosteroids, external-beam radiation therapy, surgery or systemic PCa therapy (except medical or surgical castration). This study showed a 4.1-month improvement in median OS (25.8 months in sipuleucel-T group vs. 21.7 months in placebo group) and a significant improvement in three-year survival probability $(31.7 \%$ in the sipuleucel-T group and $23.0 \%$ in the placebo group). However, there was no significant difference in the median time to objective disease progression between treatment and placebo groups (hazard ratio [HR] $0.95 ; 95 \%$ confidence interval $[\mathrm{Cl}] 0.77-1.17 ; \mathrm{p}=0.63)$, as well as clinical disease progression ( $\mathrm{HR} 0.92 ; 95 \% \mathrm{Cl}$ $0.75-1.12 ; p=0.40)$. This treatment was well-tolerated and adverse events reported more frequently in the treatment group were mostly mild to moderate and included chills, pyrexia, myalgia, and hypertension occurring within one day of infusion and resolving within 1-2 days. Only three of the 338 patients in the treatment arm were unable to receive all three infusions due to infusion-related adverse events. Sipuleucel-T was subsequently approved by the U.S. FDA (April 2010) for the treatment of patients with CRPC with asymptomatic or minimally symptomatic disease. ${ }^{28}$

\section{Treatment of $m C R P C$ with symptoms}

\section{Docetaxel}

With the development of mCRPC, patients may display symptoms that are attributable to metastatic disease burden, including bone pain, fatigue, and weight loss, a common symptom associated with PC metastases to the bone. ${ }^{9}$ As a result, these symptomatic patients displaying detectable macroscopic metastases may be referred for systemic chemotherapy, depending on performance status outside of a clinical trial. Docetaxel is a microtubule-stabilizing taxane agent administered via intravenous infusion $\left(75 \mathrm{mg} / \mathrm{m}^{2}\right.$ every three weeks) with daily oral prednisone $(5 \mathrm{mg}$ twice daily). A decade ago, docetaxel, along with prednisone, was the first agent to show significant survival benefit (2-3 months) in mCRPC, and demonstrated improved pain palliation over mitoxantrone-based (topoisomerase II-targeted) therapy in two large studies. ${ }^{29,30}$ As a result, docetaxel plus prednisone became the standard of care as first-line chemotherapy for mCRPC.

More recently, there has been promising data with regards to early introduction of docetaxel therapy in patients with high-volume metastases in conjunction with ADT. Early results of the ECOG CHAARTED (ChemoHormonal Therapy vs. Androgen Ablation Randomized Trial for Extensive Disease in Prostate Cancer) study showed a 17-month OS benefit with six cycles of docetaxel in combination with ADT vs. ADT alone (HR 0.62; $p=0.0012$ ) in patients with highvolume metastases. ${ }^{31}$ High-volume disease in this study was defined as visceral metastases or $\geq 4$ bone metastases, with $\geq 1$ bone lesion beyond the spinal column or pelvis. Median survival in low-disease-burden patients was not significant ( $H R$ 0.58; $p=0.0836)$, although this was a low sample size comprising of only $34 \%$ of the total study patients $(n=276)$.

Given the promising early results of the CHAARTED study, the current philosophy towards metastatic PCa treatment may change, as patients may receive chemotherapy prior to the development of castration resistance, which will affect subsequent treatment sequencing. Prior to this, asymptomatic metastatic PCa was often treated cautiously in hopes of minimizing ADT-related toxicity, reserving chemotherapy for patients with mCRPC. Prior to the CHAARTED study, the survival benefit of novel agents for mCRPC provided, at most, 3-5 months compared to control, paling in comparison with the survival benefit provided by early chemohormonal therapy. This study strongly encourages a shift in the treatment paradigm towards upfront chemohormonal therapy consisting of docetaxel in concert with the start of ADT, especially in men with high-volume metastatic PCa. The publication of the CHAARTED trial results are still pending.

In contrast to the CHAARTED study, an updated retrospective analysis from the GETUG-AFU 15 trial was presented 
at GU ASCO in early 2015. ${ }^{32}$ This study took place at 29 centres in France and one in Belgium, and randomized a total of 385 patients from October 2004 to December 2008 to ADT + docetaxel or ADT alone. In the study population, $58 \%$ had Gleason $\geq 8$ disease, $76 \%$ in the docetaxel arm and $67 \%$ in the ADT alone arm had metastatic disease, and median followup was 50 months. Data were analyzed based on low-volume disease (LVD) vs. high-volume disease (HVD). In the LVD group, median OS was not reached in the ADT alone group, and the median OS in the ADT + docetaxel was found to be 83.1 months. In the HVD group, a non-significant difference of four months was observed between ADT alone and ADT + docetaxel groups (35.1 vs. 39 months, HR 0.8; $p=0.35$ ). Multivariate analyses from this study showed volume of metastatic disease and alkaline phosphatase to be independent prognostic factors for OS, not treatment arm.

Differences between the CHAARTED and GETUG-AFU 15 studies may include different patient populations, with a majority of the patients in the latter study having LVD $(53 \%)$ and up to $80 \%$ of the patients in the ADT alone group receiving salvage docetaxel chemotherapy after castration resistance. The GETUG-AFU 15 study was likely also underpowered to detect a significant survival benefit in patients with HVD, with only 183 patients (91 on ADT alone vs. 92 on ADT + docetaxel). ${ }^{32}$

A much larger study was recently presented ahead of the 2015 ASCO annual meeting, which showed significant improvement in OS with the addition of docetaxel to standard hormonal therapy in men with newly diagnosed, advanced, hormone-naïve PCa. Data from the STAMPEDE trial, the largest multi-armed, randomized, PCa clinical trial conducted, showed that in 2962 patients standardized 2:1:1:1 to standard of care (SOC), SOC + docetaxel, SOC + zolendronic acid, or SOC + docetaxel + zolendronic acid, OS was approximately 77 months vs. 67 months in the SOC + docetaxel vs. SOC alone groups (HR 0.76; $p=0.003$ ). In this study, $61 \%$ of patients had metastatic disease, and all men were required to have a Gleason score of at least 8 . No statistically significant survival was seen from adding zoledronic acid. ${ }^{33}$

The STAMPEDE trial has brought further clarity in the use of docetaxel in the hormone-naïve metastatic PCa setting and strongly suggests that docetaxel chemotherapy should be offered as part of initial therapy, significantly changing the treatment algorithm for a specific subset of patients. Further study is still required to determine if there is significant survival advantage in patients with non-metastatic disease.

\section{Cabazitaxel: A novel chemotherapeutic option}

Beyond the survival benefit of a few months attained by the docetaxel-based regimen in mCRPC patients, ${ }^{29,30} \mathrm{mCRPC}$ disease progresses post-docetaxel treatment due to innate or acquired resistance, and subsequently must be treated with second-line therapeutic options. Like docetaxel, cabazitaxel is a novel taxane agent that promotes tubulin assembly and stabilizing microtubules, ${ }^{34}$ exerting cytotoxicity by blocking mitosis at the transition from metaphase to anaphase. ${ }^{35}$ Cabazitaxel is active in docetaxel-sensitive cancer models, but more importantly, it displays cytotoxicity in tumour cells that are innately resistant or have acquired resistance to docetaxel. ${ }^{36}$

Cabazitaxel was evaluated in the TROPIC study, an international, randomized, open-label phase 3 study in mCRPC patients previously treated with docetaxel. ${ }^{37}$ In this study, 755 patients were recruited from 146 centres in 26 countries and were randomized (1:1): 378 participants receiving cabazitaxel $\left(25 \mathrm{mg} / \mathrm{m}^{2}\right.$ intravenously over one hour on Day 1 of a 21-day cycle) plus oral prednisone (10 mg daily), while 377 participants received mitoxantrone $\left(12 \mathrm{mg} / \mathrm{m}^{2}\right.$ intravenously over 15-30 minutes on Day 1 of a 21 -day cycle) plus oral prednisone. The results of the study showed an OS benefit in favour of the cabazitaxel treatment group, with a median OS of 15.1 months vs. 12.7 months, and a $30 \%$ relative risk of death reduction (HR 0.70; 95\% $\mathrm{Cl} 0.59-0.83 ; \mathrm{p}<0.0001)$ compared to the mitoxantrone treatment group. Median progression-free survival (PFS) also favoured cabazitaxel (2.8 months vs. 1.4 months; HR 0.74; 95\% Cl 0.64-0.86; $\mathrm{p}<0.0001$ ), with significantly higher rates of PSA and tumour response than the mitoxantrone group. Hematological toxic effects were the most common adverse events, with greater than $88 \%$ of patients in both treatment groups affected; however, neutropenia ( $82 \%$ vs. $58 \%$ ) and febrile neutropenia $(8 \%$ vs. $1 \%$ ) were more commonly observed in the cabazitaxel group compared to mitoxantrone group, respectively. The most common non-hematological adverse effect was diarrhea in the cabazitaxel group, with $6 \%$ experiencing Grade 3 symptoms compared to $<1 \%$ in the mitoxantrone group. Moreover, there were a greater number of deaths in the cabazitaxel group $(n=18)$ within 30 days of the last dose administered compared to the mitoxantrone group $(n=9)$. Of the 18 deaths in the cabazitaxel group, seven were the consequence of neutropenia and five were from cardiac complications. Out of the nine deaths in the mitoxantrone group, six were due to PCa progression, while none of the deaths in the cabazitaxel group were due to disease progression. ${ }^{37}$

Cabazitaxel is the first cytotoxic agent demonstrated to confer an OS benefit in patients with mCRPC after docetaxel treatment. While the rate of neutropenia and febrile neutropenia were significantly higher in the cabazitaxel treatment group, the authors of the study have suggested close monitoring and management of treatment-induced symptoms. As well, primary prophylaxis with granulocyte colonystimulating factor (G-CSF) was recommended for high-risk patients (age $>65$ years and poor performance status with previous febrile neutropenia, extensive prior radiation ports, poor nutritional status, and/or the presence of other serious 
comorbidities), with dose-modification being used to mitigate the toxic effects associated with treatment. As a result, cabazitaxel was granted U.S. FDA approval (June 2010) for use in combination with prednisone for the treatment of patients with mCRPC previously treated with a docetaxelcontaining regimen, while Health Canada granted cabazitaxel (June 2011) approval as second-line chemotherapy for mCRPC patients previously treated with docetaxel.

\section{Hormonal manipulation as second-line mCRPC treatment: Novel agents that directly or indirectly target AR signaling}

As an alternative to chemotherapy-based treatment options, novel agents targeting AR signaling provide further approaches in the treatment of symptomatic mCRPC. Cytochrome p450c17 (CYP17) is an endoplasmic reticulum (ER)-localized enzyme expressed in both the testes and adrenal glands, ${ }^{38}$ which plays a critical role in the production of sex steroids and glucocorticoid from cholesterol. CYP17 catalyzes two critical steps in androgen biosynthesis through its $17 \alpha$-hydroxylase and $\mathrm{C}_{17,20}$-lyase activities. The $17 \alpha$-hydroxylase activity of CYP17 converts pregnenolone to $17 \alpha$-pregnenolone and progesterone to $17 \alpha$-progesterone. The $\mathrm{C}_{17,20}$-lyase activity of CYP17 converts $17 \alpha$-hydroxypregnenolone to dehydroepiandrosterone (DHEA) and $17 \alpha$-hydroxyprogesterone to androstenedione. ${ }^{39}$ In addition to androgen biosynthesis, CYP17 also plays a key role in glucocorticoid production, with $17 \alpha$-pregnenolone and $17 \alpha$-progesterone serving as precursors in glucocorticoid biosynthesis (Fig. 2). ${ }^{39}$ The inhibition of $17 \alpha$-hydroxylase activity dramatically reduces the production of cortisol, with low serum cortisol levels stimulating a cortisol-adrenocorticotropic hormone (ACTH) feedback loop, leading to increased serum ACTH release from the pituitary gland and subsequent excessive mineralocorticoid (aldosterone) production (Fig. 1). This clinically manifests as fluid overload, hypokalemia, renin suppression, and arterial hypertension, ${ }^{40}$ necessitating low-dose glucocorticoid supplementation along with CYP17 inhibitor treatment.

\section{Abiraterone}

Abiraterone acetate (AA) is the prodrug of abiraterone, an irreversible, highly selective CYP17 inhibitor that targets its $17 \alpha$-hydroxylase and $\mathrm{C}_{17,20}$-lyase activities (Fig. 2), ${ }^{41}$ resulting in reduced testosterone production in adrenal, testicular, and prostate tumours (Fig. 1). ${ }^{42}$ The safety, efficacy, and tolerability of AA were demonstrated in phase $1 / 2$ trials administered either alone or with oral prednisone, with significant antitumour activity being reported in MCRPC patients who had previously received chemotherapy, such as docetaxel, and in those patients who were chemotherapy-naïve. ${ }^{43-46}$ This led to further phase 3 evaluation of AA plus prednisone in both the pre- and post-docetaxel treatment setting.

The landmark COU-AA-301 trial (NCT00091442) was an international, randomized, double-blind, placebo-controlled phase 3 study of AA administered with prednisone in $\mathrm{mCRPC}$ patients previously treated with docetaxel. ${ }^{12,47}$ Eligible patients had histologically or cytologically confirmed diagnosis of $\mathrm{PCa}$, ongoing androgen deprivation with castrate levels of testosterone $(<50 \mathrm{ng} / \mathrm{dl})$, previous treatment with docetaxel, and progressive disease involving increasing PSA values or radiologically confirmed progression with or without a rise in PSA level. In this study, 1195 patients were recruited from 147 sites in 13 countries and participants were randomized (2:1) whereby 797 patients received AA (1 g orally once daily) plus prednisone (5 mg orally twice daily) and 398 patients received placebo plus prednisone. Interim analysis of the study populations revealed a median OS of 14.8 months in the AA treatment group and 10.9 months in the placebo group. ${ }^{47}$ Based on these interim results, the study data was unblinded and patients in the placebo group were switched over to AA if they met criteria for crossover treatment. Furthermore, AA was approved by the U.S. FDA and Health Canada in 2011 for use in combination with prednisone for the treatment of patients with mCRPC who have received prior docetaxel treatment. Final analysis of the COU-AA-301 trial was published in October 2012, which showed that the median OS for the AA group was longer than in the placebo group (15.8 months vs. 11.2 months; HR $0.74 ; 95 \% \mathrm{Cl} 0.64-0.86 ; \mathrm{p}<0.0001$ ) with no additional safety concerns being identified with further followup. ${ }^{12}$

The second landmark trial for AA, COU-AA-302 (NCT00887198) was an international, randomized, doubleblind, placebo-controlled phase 3 study of AA along with prednisone in chemotherapy-naïve patients with mCRPC. ${ }^{48,49}$ In this study, a total of 1088 patients from 151 centres in 12 countries were randomized in a 1:1 ratio, with 546 participants receiving AA ( $1 \mathrm{~g}$ of AA orally once daily) plus prednisone (5 mg orally twice daily) and 542 participants receiving placebo plus prednisone. The interim analysis results of this phase 3 study, presented at the ASCO 2012 conference, demonstrated a statistically significant improvement in relative PFS (rPFS) across all patient subgroups, with median rPFS in the placebo group being 8.3 months, while the median rPFS in the AA group was not reached (HR 0.43; 95\% Cl 0.35-0.52; $\mathrm{p}<0.0001) .{ }^{48,49} \mathrm{~A}$ strong trend in OS was observed in all patient subgroups, although OS did not reach statistical significance; the placebo group was found to have a median OS of 27.2 months, while the AA group was not reached (HR 0.75; 95\% Cl 0.61-0.93; $\mathrm{p}<0.0097) .{ }^{48}$ Common adverse events in the AA group included fatigue, fluid retention, hypokalemia, hypertension, and elevated aminotransferases. Based on the interim analysis of OS at $43 \%$ of death 


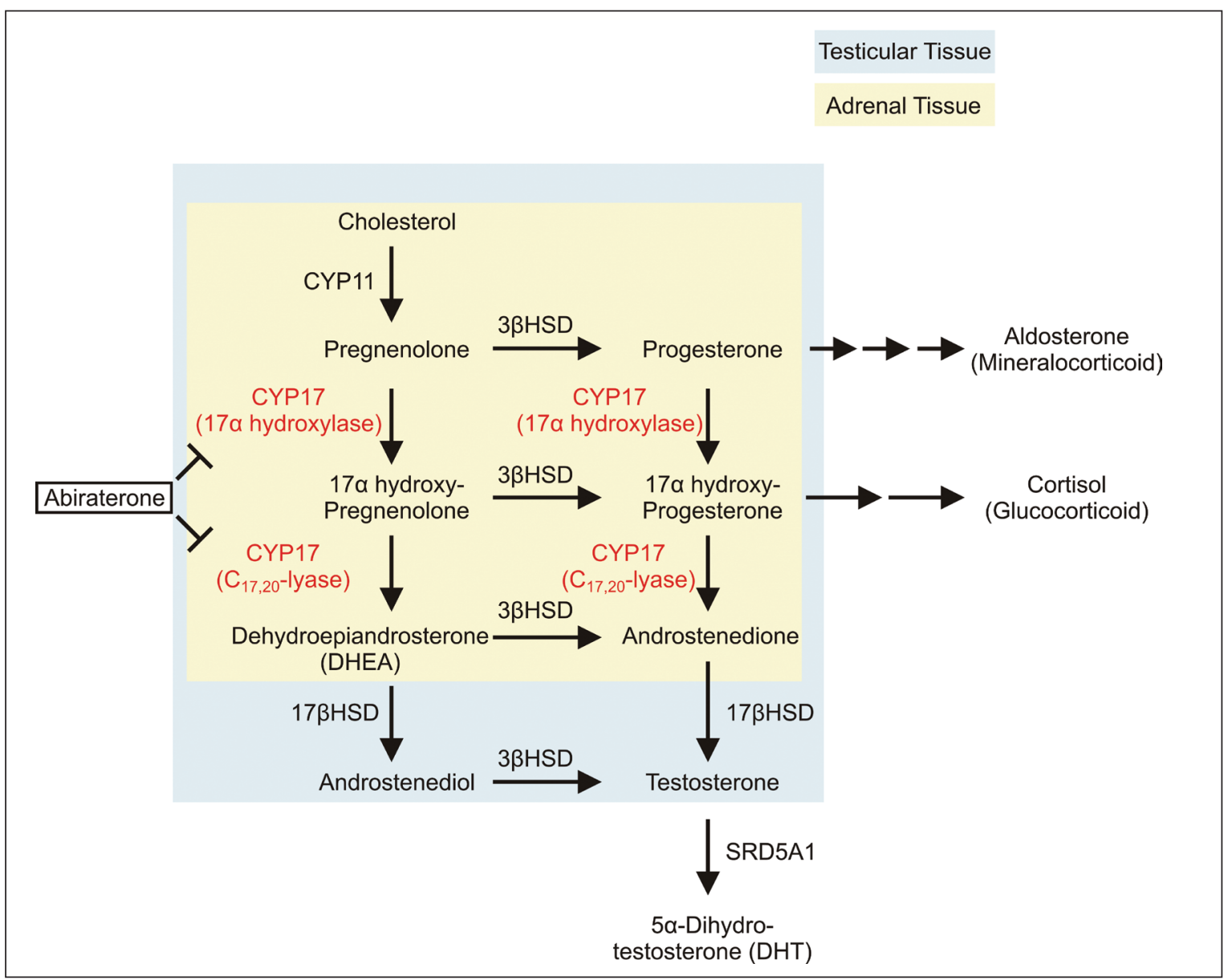

Fig 2. Ablating androgen biosynthesis as a novel treatment option for metastatic castrate-resistant prostate cancer. Androgens are synthesized from cholesterol through multiple enzymatic reactions within adrenal and testicular tissues. Cytochrome p450c17 (CYP17), an ER-localized enzyme expressed in both the testes and adrenal glands, plays a critical role in the androgen biosynthesis pathway. CYP17 catalyzes two critical steps through its $17 \alpha$-hydroxylase and C17,20-lyase activities. The $17 \alpha$-hydroxylase activity of CYP17 converts pregnenolone to $17 \alpha$-pregnenolone and progesterone to $17 \alpha$-progesterone. The C17,20-lyase activity of CYP17 converts $17 \alpha$-hydroxypregnenolone to dehydroepiandrosterone (DHEA) and 17 $\alpha$-hydroxyprogesterone to androstenedione, which are subsequently converted to testosterone and/or $5 \alpha$-dihydrotestosterone (DHT) by other steroidogenic enzymes. Abiraterone is a novel CYP17 inhibitor that acts to inhibit its 17 $\alpha$-hydroxylase and/or C17,20-lyase activities.

events (333 deaths of trial subjects), which took place in the fourth quarter of 2011 by an independent data-monitoring committee (DMC), the study was unblinded and patients from the placebo group were offered crossover therapy to AA.

Final analysis of the COU-AA-302 trial was published in February 2015. Median OS was found to be significantly longer in the abiraterone acetate group than in the placebo group (34.7 months [95\% Cl 32.7-36.8] vs. 30.3 months [95\% Cl 28.7-33.3]; HR 0.81; $p=0.0033$ ). The median followup time was 49.2 months, after $96 \%$ of the pre-specified 773 death events for final analysis had been observed. A total of $44 \%$ of the patients in the placebo group received abiraterone acetate plus prednisone as either part of the crossover protocol or as subsequent treatment. ${ }^{49}$

While the COU-AA-302 trial is one of the first phase 3 clinical trials to use rPFS as a primary endpoint in the chemotherapy-naïve PCa population, whether rPFS represents a tangible clinical benefit remains to be seen. It is also uncertain why the DMC decided to end the clinical trial prior to reaching statistical significance in OS despite a strong trend in data. Based on the interim analysis results, AA was granted U.S. FDA approval as treatment in the predocetaxel setting in December 2012.

\section{Enzalutamide: A second-generation AR antagonist}

While castration or first-generation AR antagonist treatment has been shown to effectively treat androgen-sensitive PCa prior to CRPC development, alterations in the AR ligandbinding domain promote these AR antagonists to become partial agonists in a subset of CRPC, further promoting tumour progression. ${ }^{50}$ Enzalutamide (MDV-3100) is a small molecule 
that irreversibly binds $A R,{ }^{51}$ preventing its nuclear translocation, DNA binding, and subsequent AR-driven transcription. Not only has it has also been found to have a higher affinity for the receptor than previously available AR antagonists, it has not demonstrated any AR agonistic effects. ${ }^{51}$

The AFFIRM study was an international, randomized, double-blind, placebo-controlled phase 3 study of enzalutamide in patients with $\mathrm{mCRPC}$ previously treated with docetaxel..$^{13}$ In this study, 1199 patients were recruited from 156 sites in 15 countries, randomized in a 2:1 ratio in which 800 participants received enzalutamide $(160 \mathrm{mg}$ orally once daily) while 399 participants received matched placebo capsules. Interim analysis of the study populations revealed a median OS of 18.4 months $(95 \% \mathrm{Cl} 17.3-$ not reached) in the enzalutamide group and 13.6 months (95\% $\mathrm{Cl} 11.3-15.8)$ in the placebo group. Moreover, there was a $37 \%$ reduction in the risk of death following enzalutamide as compared to placebo (HR 0.63; 95\% Cl 0.53-0.75; $\mathrm{p}<0.001)$. Based on these preliminary results, this study was halted, unblinded, and eligible patients in the placebo group were placed on enzalutamide. The OS benefit was consistent even after adjusting for stratification factors, including age, baseline pain intensity, geographic region, and disease progression at entry. Enzalutamide was also superior to placebo in the measured secondary endpoints, including PSA level response rate ( $54 \%$ vs. $2 \% ; p<0.001)$, soft tissue response rate $(29 \%$ vs. $4 \% ; p<0.001)$, functional assessment of cancer therapy-prostate (FACT-P) quality of life response (43\% vs. $18 \% ; \mathrm{p}<0.001)$, time to PSA progression (8.3 vs. 3.0 months; HR 0.25; $\mathrm{p}<0.001$ ), rPFS (8.3 vs. 2.9 months; HR 0.40; $p<0.001)$, and time to first skeletal related event (SRE) (16.7 vs. 13.3 months; HR 0.69; $\mathrm{p}<0.001)$. Adverse events reported more frequently in the enzalutamide group included fatigue, diarrhea, hot flashes, musculoskeletal pain, and headaches. In addition, five of the 800 patients $(0.96 \%)$ in the enzalutamide group were reported to have had a seizure, whereas no seizures were reported in the placebo group. This increase in seizure incidence is believed to be due to the ability of enzalutamide to cross the blood-brain barrier and inhibit GABA channels, with convulsions being observed to be dose-dependent when administered above the therapeutic range in animal studies. ${ }^{52}$ Thus, caution should be used when administering enzalutamide in patients with a known history of seizures.

Enzalutamide received U.S. FDA approval (August 2012) for patients with mCRPC previously treated with docetaxel, with a recommended dose of $160 \mathrm{mg}$ orally once daily, and will not require concomitant steroid administration. Enzalutamide received approval from Health Canada (May 2013) for the treatment of mCRPC in patients previously treated with docetaxel chemotherapy.

Based on its mechanism of action, enzalutamide should be active in patients with AR-driven mCRPC, regardless of whether or not they have previously received docetaxel, as in the AFFIRM trial. The PREVAIL (NCT01212991) trial is a randomized, double-blind, placebo-controlled, multinational phase 3 trial currently evaluating daily (160 mg) enzalutamide treatment in 872 patients compared to placebo in 845 patients with progressive mCRPC not having previously received chemotherapy. Treatment in patients continued until radiographic progression of the disease was confirmed or side effects were reported, after which chemotherapy was initiated. Interim analysis was performed at 20 months, showing a significant reduction in the risk of death by $29 \%$ (HR 0.706; $p<0.0001)$. The rPFS was $65 \%$ among enzalutamide-treated patients compared to $14 \%$ in the placebo group at 12 months followup (81\% risk reduction; HR $0.19 ; 95 \% \mathrm{Cl} 0.15-0.23 ; \mathrm{p}<0.001)$. Furthermore, the median time until the initiation of chemotherapy was 28.0 months in the enzalutamide group compared to 10.8 months in the placebo group (HR 0.35; $\mathrm{p}<0.001) .{ }^{53}$ Patients with a history of seizure disorders were excluded from this trial. U.S. FDA approval for enzalutamide as a first-line therapy for mCRPC was issued in September 2014. The use of enzalutamide in the chemotherapy-naïve setting has not yet been approved by Health Canada.

\section{Novel therapies for management of $\mathrm{mCRPC}$}

\section{Bone-targeted therapies}

Approximately $90 \%$ of mCRPC patients will present with bone metastases; these cause morbidity due to pain and SREs, such as pathologic fractures and spinal cord compression, which require surgical intervention or external beam radiotherapy to the bone. ${ }^{30}$ Furthermore, the presence of bone metastases have been shown to negatively impact survival, with less than a $50 \%$ five-year survival for such patients. ${ }^{54}$ Current bone-targeted therapies for mCRPC fall into two categories: osteoclast inhibitors and radiopharmaceuticals. Anti-resorptive agents have been extensively studied in the setting of mCRPC and are approved for bone pain palliation and SRE prevention/delay. Zoledronic acid is a bisphosphonate, an analogue of endogenous pyrophosphate found in bone, that becomes released as a result of osteoclast-mediated bone resorption, ${ }^{55}$ subsequently inducing apoptosis of osteoclasts. ${ }^{56}$ Clinical trials have shown significant delay in the time to SRE vs. placebo, with improved pain control. ${ }^{57}$ Denosumab is a monoclonal antibody to the receptor activator of NF kappaB ligand (RANKL). Binding of RANKL to its cognate receptor, RANK, on monocytes results in osteoclast differentiation and their subsequent activation of bone resorption. ${ }^{58}$ Therefore, binding of denosumab to RANKL inhibits osteoclast formation and activation, and prevents bone resorption. ${ }^{59,60}$ An international phase 3 trial 
compared denosumab to zoledronic acid for the prevention of SREs in mCRPC patients with current or a history of radiologically evident bone metastases, and demonstrated a 3.6-month improvement in time to SRE for denosumab over zoledronic acid (20.7 vs. 17.1 months, respectively; $p=0.0002) .{ }^{61}$ However, neither zoledronic acid nor denosumab have shown improvement in OS or disease progression in either study arm.

Radium-223 $\left.{ }^{223} \mathrm{Ra}\right)$ is a bone-targeting alpha-emitter with a half-life of 11.4 days. Alpha-emitters have a high linear energy transfer and short track length, with a range of less than $100 \mu \mathrm{m}$. With a short track length, it was thought that there would be relative sparing of the bone marrow, with increased localized anti-tumour effect by virtue of higher energy delivery. ${ }^{62}$ The ALpharadin in SYMPtomatic Prostate Cancer (ALSYMPCA) trial was a double-blind, randomized, international phase 3 study evaluating the efficacy of ${ }^{223} \mathrm{Ra}$ with best standards of care (BSC) compared to placebo with BSC in patients with symptomatic MCRPC and known skeletal metastases. Eligible mCRPC patients had multiple skeletal metastases ( $\geq 2$ hot spots on bone scan) with regular analgesic administration or treatment with external beam radiation therapy for bone pain. A total of 922 patients were randomized (2:1 ratio), with 615 participants receiving six IV administrations (separated in four-week intervals) of ${ }^{223} \mathrm{Ra}$ at $50 \mathrm{kBq} / \mathrm{kg}$ body weight and 307 receiving placebo. Planned interim analysis once 314 deaths had occurred showed that ${ }^{223} \mathrm{Ra}$ significantly improved OS vs. placebo, with a median OS of 14.0 months vs. 11.2 months, respectively (HR 0.695; $95 \% \mathrm{Cl} 0.552-0.875 ; \mathrm{p}=0.00185)$. An updated analysis at 528 deaths confirmed survival benefit with ${ }^{223} \mathrm{Ra}$, with median OS of 14.9 months vs. 11.3 months for placebo (HR $0.70 ; 95 \% \mathrm{Cl}, 0.58-0.83 ; \mathrm{p}<0.001)$. The safety profile and tolerability of ${ }^{223}$ Ra was favourable, showing low incidence of myelosuppression. ${ }^{63}$

As a result, ${ }^{223} \mathrm{Ra}$ is the first bone-targeted therapy in mCRPC with skeletal metastases to show a survival benefit. Unlike recent U.S. FDA-approved treatments for CRPC, such as cabazitaxel, AA, and enzalutamide, the ALSYMPCA trial included and showed OS benefit in patients who were pre-docetaxel and may not have been able to tolerate cytotoxic chemotherapy for CRPC treatment. Given its favourable safety profile and tolerability, ${ }^{223}$ Ra may be a potential treatment for CRPC patients with bone metastases who are docetaxel-naive. Based on the results of the interim analysis of the ALSYMPCA trial, ${ }^{63}{ }^{223} \mathrm{Ra}$ has been approved by the U.S. FDA (May 2013) and Health Canada (February 2015) to treat men with symptomatic late-stage $\mathrm{MCRPC}$ with isolated bone metastasis.

\section{Conclusion}

The landscape for the treatment of mCRPC is quickly changing, with the development of therapies targeting further hor- monal manipulation (AA and enzalutamide), PCa metastases to the bone $\left({ }^{223} \mathrm{Ra}\right)$, and cytotoxic chemotherapies (docetaxel and cabazitaxel) showing OS benefit in phase 3 clinical trials. Within the past two years, the positive results of the PREVAIL trial have further opened up the use of enzalutamide in the pre-docetaxel setting. More recently, abiraterone has been shown to be converted by $3 \beta$-hydroxysteroid dehydrogenase $(3 \beta \mathrm{HSD})$ isoenzymes to the more active molecule, $\Delta^{4}$-abiraterone, which inhibits the androgen receptor and multiple steroidogenic enzymes (CYP17, 3 $\beta \mathrm{HSD}$, and SRD5A) and demonstrates more potent tumour-inhibiting activity than abiraterone in the preclinical setting. ${ }^{64}$ Whether $\Delta^{4}$-abiraterone will be more effective than AA treatment in the clinical setting is anticipated to be addressed in the foreseeable future. ${ }^{64}$

Unlike AA, enzalutamide does not interfere with androgen production, but rather inhibits AR signaling directly. As AA requires Co-administration with glucocorticoids to curtail side effects, preclinical studies have shown that disease progression following abiraterone may occur due to glucocorticoid-induced AR signal activation, which may be inhibited by enzalutamide treatment; ${ }^{65}$ this provides strong rationale for the clinical evaluation of CYP17 inhibition in combination with AR antagonism. However, caution should be taken, as a recent preclinical study suggests that glucocorticoid receptor expression increases and binds to $\sim 50 \%$ of all AR binding sites in enzalutamide-resistant PCa cells, bypassing AR blockade, ${ }^{66}$ which may render such a combinatorial therapy ineffective. Therefore, effective combinatorial treatment or sequential administration of these novel agents will require close monitoring of tumours during treatment in order to achieve an effective response in mCRPC-targeted therapy.

The treatment paradigm separating $\mathrm{mCRPC}$ treatments into pre-docetaxel and post-docetaxel settings are largely artificial, based on the fact that docetaxel was the first agent to demonstrate OS benefit in the setting of mCRPC. As mentioned, further phase 3 clinical trials have demonstrated OS benefit prior to docetaxel therapy for both enzalutamide and AA, making all three agents possible first-line treatments for mCRPC. ${ }^{223}$ Ra treatment has also shown evidence of OS benefit in the pre-docetaxel therapy setting with largely bone metastases. The proper sequencing of these agents will become increasingly important as patients show progression on different agents, and more studies are needed to determine the best treatments post-enzalutamide, postAA, or following ${ }^{223} \mathrm{Ra}$. There is also ongoing research on new mechanisms of $A R$ resistance to these new agents. For example, splice variants of $A R$, such as AR-V7, have demonstrated enzalutamide resistance. ${ }^{67}$ The ability to screen patients for these mechanisms of therapy resistance may further support and guide optimal therapeutic decision-making. ${ }^{68}$ Furthermore, a promising study has recently shown that cabazitaxel remains active as a third-line agent even 
after first-line docetaxel and second-line AA treatment. ${ }^{69}$ Taken into consideration with the results of the CHAARTED trial, in which early docetaxel administration was shown to significantly improve OS in combination with ADT in the setting of non-mCRPC, cabazitaxel may play a much more central role in the management of mCRPC.

In the coming years, it will be of paramount importance for urologists to have a sound understanding of the hormonal, systemic, and bone-targeted therapies available for the treatment of PCa. As primary physicians in both the U.S. and Canada continue their efforts to resist PSA screening for $\mathrm{PCa}$, it is likely that men presenting to the urologist with $\mathrm{PCa}$ in the metastatic state will be a much more common occurrence. Being familiar with the treatment options available and collaborating with medical oncologists will improve the delivery of care to patients with mCRPC.

Competing interests: The authors report no competing personal or financial interests.

Acknowledgements: This work was supported by research funding from the McMaster Institute of Urology and St. Joseph's Hospital (Hamilton, ON, Canada).

This paper has been peer-reviewed.

\section{References}

1. Canadian Cancer Society. Prostate Cancer Statistics, 2014. Prostate Cancer Stat 2015.

2. Siegel R, Ma J, Zou Z, et al. Cancer statistics, 2014. CA Cancer J Clin 2014;64:9-29. http://dx.doi. org/10.3322/caac.21208

3. Ross JS. The androgen receptor in prostate cancer: Therapy target in search of an integrated diagnostic test. Adv Anat Pathol 2007;14:353-7. http://dx.doi.org/10.1097/PAP.0b013e31814a52c4

4. Moon C, Park JC, Chae YK, et al. Current status of experimental therapeutics for prostate cancer. Cancer Lett 2008;266:116-34. http://dx.doi.org/10.1016/i.canlet.2008.02.065

5. Huggins $C$, Hodges C V. Studies on prostatic cancer. I. The effect of castration, of estrogen and androgen injection on serum phosphatases in metastatic carcinoma of the prostate. CA Cancer I Clin 1972;22:23240. http://dx.doi.org/10.3322/caniclin.22.4.232

6. Gomella LG. Effective testosterone suppression for prostate cancer: Is there a best castration therapy? Rev Urol 2009;11:52-60.

7. Crawford ED, Eisenberger MA, McLeod DG, et al. A controlled trial of leuprolide with and without flutamide in prostatic carcinoma. N Engl J Med 1989;321:419-24. http://dx.doi.org/10.1056/ NEJM198908173210702

8. Eisenberger MA, Blumenstein BA, Crawford ED, et al. Bilateral orchiectomy with or without flutamide for metastatic prostate cancer. N Engl J Med 1998;339:1036-42. http://dx.doi.org/10.1056/ NEJM199810083391504

9. Cookson MS, Roth BJ, Dahm P, et al. Castration-resistant prostate cancer: AUA guideline. J Urol 2013;190:429-38. http://dx.doi.org/10.1016/i.juro.2013.05.005

10. Saad F, Chi KN, Finelli A, et al. The 2015 CUA-CUOG guidelines for the management of castration-resistant prostate cancer (CRPC). Can Urol Assoc J 2015;9:90-6. http://dx.doi.org/10.5489/cuaj.2526

11. Small EJ, Halabi S, Dawson NA, et al. Antiandrogen withdrawal alone or in combination with ketoconazole in androgen-independent prostate cancer patients: A phase 3 trial (CALGB 9583). J Clin Oncol 2004;22:1025-33. http://dx.doi.org/10.1200/JC0.2004.06.037

12. Fizazi K, Scher HI, Molina A, et al. Abiraterone acetate for treatment of metastatic castration-resistant prostate cancer: Final overall survival analysis of the COU-AA-301 randomized, double-blind, placebo-controlled phase 3 study. Lancet Oncol 2012;13:983-92. http://dx.doi.org/10.1016/S1470-2045(12)70379-0

13. Scher HI, Fizazi K, Saad F, et al. Increased survival with enzalutamide in prostate cancer after chemotherapy. N Engl J Med 2012;367:1187-97. http://dx.doi.org/10.1056/NEJMoal207506
14. Locke JA, Guns ES, Lubik AA, et al. Androgen levels increase by intra-tumoural de novo steroidogenesis during progression of castration-resistant prostate cancer. Cancer Res 2008;68:6407-15. http://dx.doi. org/10.1158/0008-5472.CAN-07-5997

15. Titus $M A$, Schell MJ, Lih $F B$, et al. Testosterone and dihydrotestosterone tissue levels in recurrent prostate cancer. Clin Cancer Res 2005;11:4653-7. http://dx.doi.org/10.1158/1078-0432.CCR-05-0525

16. Taylor BS, Schultz N, Hieronymus H, et al. Integrative genomic profiling of human prostate cancer. Cancer Cell 2010;18:11-22. hittp://dx.doi.org/10.1016/i.ccr.2010.05.026

17. Linja MJ, Savinainen KJ, Saramaki OR, et al. Amplification and overexpression of androgen receptor gene in hormone-refractory prostate cancer. Cancer Res 2001;61:3550-5.

18. Taplin ME, Bubley GJ, Ko YJ, et al. Selection for androgen receptor mutations in prostate cancers treated with androgen antagonist. Cancer Res 1999;59:2511-5.

19. Coffey K, Robson CN. Regulation of the androgen receptor by post-translational modifications. J Endocrinol 2012;215:221-37. http://dx.doi.org/10.1530/JOE-12-0238

20. Drake JM, Graham NA, Stoyanova T, et al. Oncogene-specific activation of tyrosine kinase networks during prostate cancer progression. Proc Natl Acad Sci U S A 2012;109:1643-8. http://dx.doi.org/10.1073/ pnas. 1120985109

21. Rybak AP, Bristow RG, Kapoor A. Prostate cancer stem cells: Deciphering the origins and pathways involved in prostate tumorigenesis and aggression. Oncotarget 2015;6:1900-19. http://dx.doi.org/10.18632/ oncotarget.2953

22. Mezynski J, Pezaro C, Bianchini D, et al. Anti-tumour activity of docetaxel following treatment with the CYP17A1 inhibitor abiraterone: Clinical evidence for cross-resistance? Ann Oncol 2012;23:2943-7. http://dx.doi.org/10.1093/annonc/mds119

23. Noonan KL, North S, Bitting RL, et al. Clinical activity of abiraterone acetate in patients with metastatic castration-resistant prostate cancer progressing after enzalutamide. Ann Oncol 2013;24:1802-7. http://dx.doi.org/10.1093/annonc/mdt138

24. Pezaro CJ, Omlin AG, Altavilla A, et al. Activity of cabazitaxel in castration-resistant prostate cancer progressing after docetaxel and next-generation endocrine agents. Eur Urol 2014;66:459-65. http://dx.doi.org/10.1016/i.eururo.2013.11.044

25. Small EJ, Schellhammer PF, Higano CS, et al. Placebo-controlled phase 3 trial of immunologic therapy with sipuleucel-T (APC8015) in patients with metastatic, asymptomatic hormone refractory prostate cancer. J Clin Oncol 2006;24:3089-94. http://dx.doi.org/10.1200/JC0.2005.04.5252

26. Higano CS, Schellhammer PF, Small EJ, et al. Integrated data from 2 randomized, double-blind, placebocontrolled, phase 3 trials of active cellular immunotherapy with sipuleucel-T in advanced prostate cancer. Cancer 2009;115:3670-9. http://dx.doi.org/10.1002/cncr.24429

27. Small EJ, Fratesi P, Reese DM, et al. Immunotherapy of hormone-refractory prostate cancer with antigenloaded dendritic cells. J Clin Oncol 2000;18:3894-903.

28. Kantoff PW, Higano CS, Shore ND, et al. Sipuleucel-T immunotherapy for castration-resistant prostate cancer. N Engl J Med 2010;363:411-22. http://dx.doi.org/10.1056/NEJMoa1001294

29. Tannock IF, de Wit R, Berry WR, et al. Docetaxel plus prednisone or mitoxantrone plus prednisone for advanced prostate cancer. N Engl J Med 2004;351:1502-12. http://dx.doi.org/10.1056/ NEJMoa040720

30. Petrylak DP, Tangen CM, Hussain MHA, et al. Docetaxel and estramustine compared with mitoxantrone and prednisone for advanced refractory prostate cancer. N Engl J Med 2004;351:1513-20. http:// dx.doi.org/10.1056/NEJMoa041318

31. Sweeney CJ, Chen Y-H, Carducci M, et al. Chemohormonal therapy in metastatic hormone-sensitive prostate cancer. N Engl J Med 2015;373:737-46. http://dx.doi.org/10.1056/NEJMoa1503747

32. Gravis $G$, Boher J-M, Joly F, et al. Androgen deprivation therapy (ADT) plus docetaxel vs. ADT alone in metastatic non-castrate prostate cancer: Impact of metastatic burden and long-term survival analysis of the randomized phase 3 GETUG-AFU15 trial. Eur Urol 2016;70:256-62. htp://dx.doi.org/10.1016/i.eururo.2015.11.005

33. James ND, Spears MR, Clarke NW, et al. Survival with newly diagnosed metastatic prostate cancer in the "docetaxel era": Data from 917 patients in the control arm of the STAMPEDE trial (MRC PR08, CRUK/06/019). Eur Urol 2015;67:1028-38. http://dx.doi.org/10.1016/i.eururo.2014.09.032

34. Garcia $\mathrm{P}$, Braguer $D$, Carles $G$, et al. Comparative effects of taxol and taxotere on two different human carcinoma cell lines. Cancer Chemother Pharmacol 1994;34:335-43. http://dx.doi.org/10.1007/BF00686042

35. Wilson L, Jordan MA. New microtubule/tubulin-targeted anticancer drugs and novel chemotherapeutic strategies. J Chemother 2004;16 Suppl 4:83-5. http://dx.doi.org/10.1179/joc.2004.16.Supplement-1.83

36. Vrignaud $P$, Semiond D, Lejeune $P$, et al. Preclinical anti-tumour activity of cabazitaxel, a semisynthetic taxane active in taxane-resistant tumours. Clin Cancer Res 2013;19:2973-83. http://dx.doi. org/10.1158/1078-0432.CCR-12-3146

37. de Bono IS, Oudard S, Ozguroglu M, et al. Prednisone plus cabazitaxel or mitoxantrone for metastatic castration-resistant prostate cancer progressing after docetaxel treatment: A randomized open-label trial. Lancet (London, England) 2010;376:1147-54. http://dx.doi.org/10.1016/50140-6736(10)61389-X

38. Brock BJ, Waterman MR. Biochemical differences between rat and human cytochrome P450c17 support the different steroidogenic needs of these two species. Biochemistry 1999;38:1598-606. http://dx.doi. org/10.1021/bi9821059 
39. Salvador JAR, Pinto RMA, Silvestre SM. Steroidal 5alpha-reductase and 17alpha-hydroxylase/17,20lyase (CYP17) inhibitors useful in the treatment of prostatic diseases. J Steroid Biochem Mol Biol 2013;137:199-222. http://dx.doi.org/10.1016/i.jsbmb.2013.04.006

40. Fluck CE. Congenital adrenal hyperplasia owing to 17 alpha-hydroxylase $/ 17,20$ lyase and $p 450$ oxidoreductase deficiencies. Adv Exp Med Biol 2011;707:3-5. http://dx.doi.org/10.1007/978-1-44198002-1_1

41. Logothetis CJ, Efstathiou E, Manuguid F, et al. Abiraterone acetate. Nat Rev Drug Discov 2011;10:573-4. http://dx.doi.org/10.1038/nrd3516

42. Jagusch C, Negri M, Hille UE, et al. Synthesis, biological evaluation and molecular modelling studies of methyleneimidazole substituted biaryls as inhibitors of human 17alpha-hydroxylase-17,20-1yase (CYP17). Part I: Heterocyclic modifications of the core structure. Bioorg Med Chem 2008;16:1992-2010. http:// dx.doi.org/10.1016/j.bmc.2007.10.094

43. Attard G, Reid AHM, Yap TA, et al. Phase 1 clinical trial of a selective inhibitor of CYP17, abiraterone acetate, confirms that castration-resistant prostate cancer commonly remains hormone driven. J Clin Oncol 2008;26:4563-71. http://dx.doi.org/10.1200/JC0.2007.15.9749

44. Ryan CJ, Smith MR, Fong L, et al. Phase 1 clinical trial of the CYP17 inhibitor abiraterone acetate demonstrating clinical activity in patients with castration-resistant prostate cancer who received prio ketoconazole therapy. J Clin Oncol 2010;28:1481-8. hittp://dx.doi.org/10.1200/JC0.2009.24.1281

45. Danila DC, Morris MJ, de Bono IS, et al. Phase 2 multicentre study of abiraterone acetate plus prednisone therapy in patients with docetaxel-treated castration-resistant prostate cancer. J Clin Oncol 2010;28:1496501. http://dx.doi.org/10.1200/JC0.2009.25.9259

46. Ryan CJ, Shah S, Efstathiou E, et al. Phase 2 study of abiraterone acetate in chemotherapy-naive metastatic castration-resistant prostate cancer displaying bone flare discordant with serologic response. Clin Cancer Res 2011;17:4854-61. http://dx.doi.org/10.1158/1078-0432.CCR-11-0815

47. de Bono JS, Logothetis $\mathrm{CJ}$, Molina A, et al. Abiraterone and increased survival in metastatic prostate cancer. N Engl J Med 2011;364:1995-2005. http://dx.doi.org/10.1056/NEJMoa1014618

48. Ryan CJ, Smith MR, de Bono JS, et al. Abiraterone in metastatic prostate cancer without previous chemotherapy. N Engl J Med 2013;368:138-48. http://dx.doi.org/10.1056/NEJMoal209096

49. Ryan CJ, Smith MR, Fizazi K, et al. Abiraterone acetate plus prednisone vs. placebo plus prednisone in chemotherapy-naive men with metastatic castration-resistant prostate cancer (COU-AA-302): Final overall survival analysis of a randomized, double-blind, placebo-controlled phase 3 study. Lancet Oncol 2015;16:152-60. http://dx.doi.org/10.1016/S1470-2045(14)71205-7

50. Chen CD, Welsbie DS, Tran C, et al. Molecular determinants of resistance to antiandrogen therapy. Nat Med 2004;10:33-9. http://dx.doi.org/10.1038/nm972

51. Jung ME, Ouk S, Yoo D, et al. Structure-activity relationship for thiohydantoin androgen receptor antagonists for castration-resistant prostate cancer (CRPC). J Med Chem 2010;53:2779-96. http://dx.doi. org $/ 10.1021 /$ im $901488 \mathrm{~g}$

52. Foster WR, Car BD, Shi H, et al. Drug safety is a barrier to the discovery and development of new androgen receptor antagonists. Prostate 2011;71:480-8. http://dx.doi.org/10.1002/pros.21263

53. Beer TM, Armstrong AJ, Rathkopf DE, et al. Enzalutamide in metastatic prostate cancer before chemotherapy. N Engl J Med 2014;371:424-33. htrp://dx.doi.org/10.1056/NEJMoa1405095

54. DePuy V, Anstrom KJ, Castel LD, et al. Effects of skeletal morbidities on longitudinal patientreported outcomes and survival in patients with metastatic prostate cancer. Support Care Cancer Off J Multinat Assoc Support Care Cancer 2007;15:869-76. http://dx.doi.org/10.1007/s00520-006-0203-x
55. Oades GM, Coxon J, Colston KW. The potential role of bisphosphonates in prostate cancer. Prostate Cancer Prostatic Dis 2002;5:264-72. http://dx.doi.org/10.1038/si.pcan.4500607

56. Hughes DE, Wright KR, Uy HL, et al. Bisphosphonates promote apoptosis in murine osteoclasts in vitro and in vivo. J Bone Miner Res 1995;10:1478-87. http://dx.doi.org/10.1002/ibmr.5650101008

57. Saad F, Gleason DM, Murray R, et al. A randomized, placebo-controlled trial of zoledronic acid in patients with hormone-refractory metastatic prostate carcinoma. J Natl Cancer Inst 2002;94:1458-68. http:// dx.doi.org/10.1093/inci/94.19.1458

58. Boyle WJ, Simonet WS, Lacey DL. Osteoclast differentiation and activation. Nature 2003;423:337-42. http://dx.doi.org/10.1038/nature01658

59. Kostenuik PJ, Nguyen HQ, McCabe J, et al. Denosumab, a fully human monoclonal antibody to RANKL, inhibits bone resorption and increases BMD in knock-in mice that express chimeric (murine/human) RANKL. J Bone Miner Res 2009;24:182-95. http://dx.doi.org/10.1359/ibmr.081112

60. Rizzoli R, Yasothan U, Kirkpatrick P. Denosumab. Nat Rev Drug Discov 2010;9:591-2. http://dx.doi. org/10.1038/nrd3244

61. Fizazi $K$, Carducci $M$, Smith $M$, et al. Denosumab vs. zoledronic acid for treatment of bone metastases in men with castration-resistant prostate cancer: A randomized, double-blind study. Lancet (London, England) 2011;377:813-22. http://dx.doi.org/10.1016/S0140-6736(10)62344-6

62. Bruland OS, Nilsson S, Fisher DR, et al. High-linear energy transfer irradiation targeted to skeletal metastases by the alpha-emitter 223Ra: Adjuvant or alternative to conventional modalities? Clin Cancer Res 2006; 12:6250s-7s. http://dx.doi.org/10.1158/1078-0432.CCR-06-0841

63. Parker C, Nilsson S, Heinrich D, et al. Alpha emitter radium-223 and survival in metastatic prostate cancer. N Engl J Med 2013;369:213-23. http://dx.doi.org/10.1056/NEJMoa1213755

64. Li Z, Bishop AC, Alyamani $M$, et al. Conversion of abiraterone to D4A drives anti-tumour activity in prostate cancer. Nature 2015;523:347-51. http://dx.doi.org/10.1038/nature14406

65. Richards J, Lim AC, Hay CW, et al. Interactions of abiraterone, eplerenone, and prednisolone with wildtype and mutant androgen receptor: A rationale for increasing abiraterone exposure or combining with MDV3100. Cancer Res 2012;72:2176-82. hitp://dx.doi.org/10.1158/0008-5472.CAN-11-3980

66. Arora VK, Schenkein E, Murali R, et al. Glucocorticoid receptor confers resistance to antiandrogens by bypassing androgen receptor blockade. Cell 2013;155:1309-22. http://dx.doi.org/10.1016/i. cell.2013.11.012

67. Antonarakis ES, Lu C, Wang H, et al. AR-V7 and resistance to enzalutamide and abiraterone in prostate cancer. N Eng/ J Med 2014;371:1028-38. http://dx.doi.org/10.1056/NEJMoal315815

68. Li Y, Chan SC, Brand $\mathrm{U}$, et al. Androgen receptor splice variants mediate enzalutamide resistance in castration-resistant prostate cancer cell lines. Cancer Res 2013;73:483-9. http://dx.doi.org/10.1158/00085472.CAN-12-3630

69. Al Nakouzi N, Le Moulec S, Albiges L, et al. Cabazitaxel remains active in patients progressing after docetaxel followed by novel androgen receptor pathway-targeted therapies. Eur Urol 2015;68:228-35. http://dx.doi.org/10.1016/i.eururo.2014.04.015

Correspondence: Dr. Anil Kapoor, McMaster Institute of Urology, St. Joseph's Hospital, Hamilton, ON, Canada; akapoor@mcmaster.co

\section{To answer the three multiple choice questions associated with this review article, go to:}

https://www.qzzr.com/c/quiz/288576/want-to-obtain-more-section-3-cpd-credits.

This program is an Accredited Self-Assessment Program (Section 3) as defined by the Maintenance of Certification Program of The Royal College of Physicians \& Surgeons of Canada, and approved by the Canadian Urological Association. Remember to visit MAINPORT (www.mainport.org/mainport/) to record your learning and outcomes. You may claim a maximum of 1 hour. 